\title{
Energy Tank-Based Wrench/Impedance Control of a Fully-Actuated Hexarotor: A Geometric Port-Hamiltonian Approach
}

\author{
Ramy Rashad, Johan B.C. Engelen and Stefano Stramigioli
}

\begin{abstract}
In this work, we show how the interactive behavior of an aerial robot can be modeled and controlled effectively and elegantly in the port-Hamiltonian framework. We present an observer-based wrench/impedance controller for a fullyactuated hexarotor. The analysis and control are performed in a geometrically consistent manner on the configuration manifold of the special Euclidean group $S E(3)$ such that the UAV's nonlinear geometric structure is exploited. The controller uses a wrench observer to estimate the interaction wrench without the use of a force/torque sensor. Moreover, the concept of energy tanks is used to guarantee the system's overall contact stability to arbitrary passive environments. The reliability and robustness of the proposed approach is validated through simulation and experiment.
\end{abstract}

\section{INTRODUCTION}

Aerial robots have attracted the attention of many research communities and industrial companies in the past two decades. Most of the current operational applications of aerial robots are restricted to passive tasks like visual inspection, surveillance, and remote sensing. Current research activities are directed towards interactive flying robots engaging in active tasks like contact-based inspection, maintenance, and manipulation. In these aerial interaction applications, the controlled Unmanned Aerial Vehicle (UAV) interacts mechanically with an unknown unstructured environment.

In the aerial robotics literature, there have been several studies on the use of fully-actuated UAVs for aerial interaction in [1]-[4]. Compared to other approaches for aerial interaction, like UAV/manipulator systems [5], [6], a fullyactuated UAV is mechanically less complex and straightforward to control since it is considered as a flying end-effector.

In the work of [1], the interaction problem was approached by an admittance control technique with a wrench observer for estimating the interaction wrench. In the work of [2], [3], a hybrid pose/wrench control technique was used, which in general requires an accurate model of the environment in addition to suffering from the problem of geometric inconsistency [7]. In the work of [4], a pure motion controller was used for the interaction which also requires an accurate model of both the aerial robot and the environment.

For the aforementioned reasons, a more suitable paradigm for interaction control is to control the interactive behavior

This work has been funded by the cooperation program INTERREG Deutschland-Nederland as part of the SPECTORS project number 143081.

R. Rashad and J. Engelen are with the Robotics and Mechatronics group, University of Twente, Enschede, The Netherlands. Email: $\{$ r.a.m.rashadhashem, j.b.c.engelen\}@utwente.nl

S. Stramigioli is with the Robotics and Mechatronics group, University of Twente, and ITMO University, Saint Petersburg, Russia. Email: s.stramigioli@utwente.nl

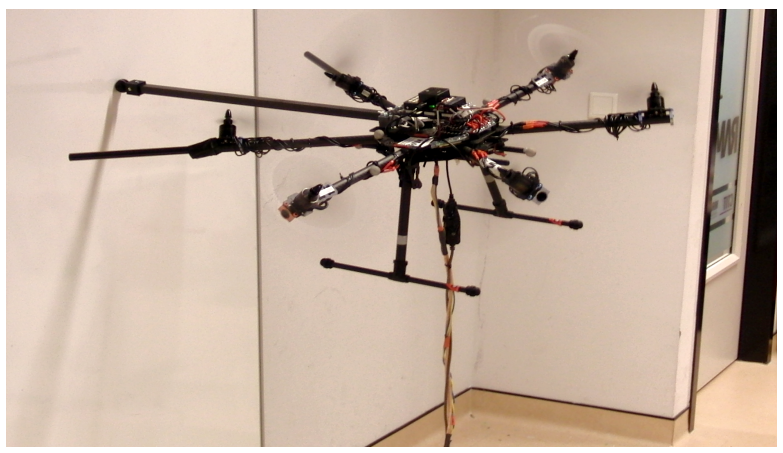

Fig. 1: Fully-actuated hexarotor with a non-zero roll angle applying a desired force to a vertical surface.

of the robot instead of the position/force independently. In this context, impedance and admittance control are among the most popular techniques that have been widely applied. In impedance and admittance control, the desired interaction wrench (force and torque) is applied using virtual displacements. However, the precise application of this desired wrench requires the knowledge of the contact surface geometrical and mechanical properties. Recently, Schindlbeck and Haddadin [8], proposed an extension to impedance control of rigid manipulators for regulating/tracking a desired interaction force. Their proposed passivity-based controller is based on energy-tanks, used to passify the non-passive action of the force tracking controller..

The concept of energy tanks has been often used in various sub-domains of robotics, e.g. bilateral telemanipulation [9], [10] and impedance control [11]-[16]. Energy tanks, first proposed in [17], are an example of the wider concept of control by energy-routing [18] and energy-aware robotics [19]. The interaction behavior of an aerial robot can be modeled effectively by the concept of power ports, which can be effectively and elegantly modeled by port-Hamiltonian systems theory. In this paradigm the control system of a robot is no longer perceived as a signal processor, but a (physical port-Hamiltonian) system connected to the aerial robot via power ports.

In this paper, we present an energy tank-based wrench/impedance controller of a fully-actuated hexarotor UAV. The modeling, analysis, and control is achieved in the port-Hamiltonian framework. The concepts considered are also presented in a geometrically consistent manner by expressing the dynamics of UAVs globally on the configuration manifold of the special Euclidean group $S E(3)$. Most closely related works to our approach are [1], [8]. 


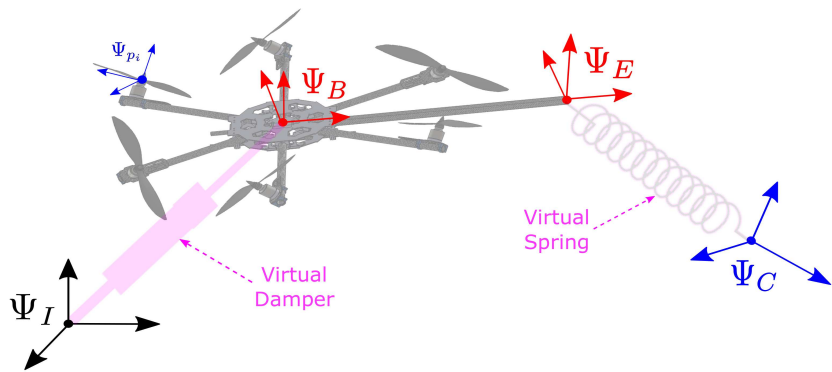

Fig. 2: Schematic view of the reference frames used and a visualization of the impedance controller.

Compared to [1], our approach is geometrically consistent and capable of regulating the interaction wrench applied to the environment. Compared to [8], the interaction in our work is achieved without external force/torque sensing by the use of wrench observers (similar to [1]). The dynamics of the wrench observer is included in the proposed control system, which has been shown to yield unstable behavior if it is not considered [8]. In the next section, we present the geometric modeling of the aerial robot followed by the control system design.

\section{UAV DYNAMIC MODELING}

\section{A. UAV Description}

A conventional hexarotor consists of six parallel propellers placed at the vertices of a planar hexagon. To modify a traditional hexarotor to be fully-actuated, the six rotors are tilted by a fixed angle, similar to Ref. [1]. Each rotor's orientation is fixed and parametrized by one fixed angle $\left(\alpha_{i}\right)$, as shown in Fig. 2.

Let $\left\{\Psi_{I}: o_{I}, \hat{\boldsymbol{x}}_{I}, \hat{\boldsymbol{y}}_{I}, \hat{\boldsymbol{z}}_{I}\right\}$ denote a right-handed orthonormal inertial frame and $\left\{\Psi_{B}: o_{B}, \hat{\boldsymbol{x}}_{B}, \hat{\boldsymbol{y}}_{B}, \hat{\boldsymbol{z}}_{B}\right\}$ denote a body-fixed frame attached to the UAV's center of mass $(\mathrm{CoM})$ and aligned with the principal inertia axes of the UAV, as shown in Fig. 2. Let $\left\{\Psi_{p_{i}}: o_{p_{i}}, \hat{\boldsymbol{x}}_{p_{i}}, \hat{\boldsymbol{y}}_{p_{i}}, \hat{\boldsymbol{z}}_{p_{i}}\right\}$ denote the frame associated with the $i$-th rotor, where $\hat{z}_{p_{i}}$ is the direction of generated thrust. The attaching location of the $i$-th propeller, for $i \in \mathcal{N}_{p}:=\{1, \cdots, 6\}$ in $\Psi_{B}$ is given by

$$
\boldsymbol{\xi}_{i}:=\boldsymbol{R}_{z}\left(\psi_{i}\right)[L, 0,0]^{\top},
$$

where $\boldsymbol{R}_{z}(\cdot) \in S O(3)$ is a rotation matrix about the $z$ axis, $L$ is the distance from the hexarotor's central axis $\hat{\boldsymbol{z}}_{B}$ to each rotor, and the angle $\psi_{i}:=(i-1) \frac{\pi}{3}$. The orientation of $\Psi_{p_{i}}$ with respect to $\Psi_{B}$ is given by

$$
\boldsymbol{R}_{p_{i}}^{B}=\boldsymbol{R}_{z}\left(\psi_{i}\right) \boldsymbol{R}_{x}\left(\alpha_{i}\right), \quad i \in \mathcal{N}_{p},
$$

where the angle $\alpha_{i}$ uniquely define the direction of the thrust generation axis $\hat{z}_{p_{i}}$ in $\Psi_{B}$. For the fully-actuated hexarotor considered in this work, we constrain ${ }^{1} \alpha_{i}=(-1)^{i+1} \alpha^{*}$, where $\alpha^{*}=47^{\circ}$. The thrust magnitude generated by the $i$ th propeller in $\Psi_{p_{i}}$ will be denoted by $\lambda_{i} \in \mathbb{R}^{+}$, while the drag torque will be expressed as $\tau_{d, i}=\gamma \sigma_{i} \lambda_{i}$, where $\gamma$ is

\footnotetext{
${ }^{1}$ This specific tilting angle is a result of an optimization-based design that will be published in a future work of the authors.
}

the propeller-specific drag-to-thrust ratio, and $\sigma_{i} \in\{-1,1\}$ specifies the direction of the propeller's rotation.

From the aforementioned definitions, the cumulative control wrench in $\Psi_{B}$ can be written as

$$
\boldsymbol{W}_{c}^{B}=\left(\begin{array}{c}
\boldsymbol{\tau}_{c}^{B} \\
\boldsymbol{f}_{c}^{B}
\end{array}\right)=\sum_{i} \lambda_{i}\left(\begin{array}{c}
\boldsymbol{\xi}_{i} \wedge \boldsymbol{u}_{i}+\gamma \sigma_{i} \boldsymbol{u}_{i} \\
\boldsymbol{u}_{i}
\end{array}\right)=: \boldsymbol{M} \boldsymbol{\lambda}
$$

where $\wedge$ denotes the vector product in $\mathbb{R}^{3}, \boldsymbol{u}_{i}:=\boldsymbol{R}_{p_{i}}^{B} \hat{\boldsymbol{e}}_{3}$ denotes the thrust generation direction of the $i$-th propeller, and $\hat{\boldsymbol{e}}_{j}$ is a vector of zeros with one at the $j$-th element, $\boldsymbol{\lambda}=\left[\lambda_{1}, \cdots, \lambda_{6}\right]^{\top}$.

\section{B. Dynamic Model}

The dynamic modeling of the fully actuated hexarotor is approached by considering it as a rigid body in the special Euclidean group $S E(3):=S O(3) \ltimes \mathbb{R}^{3}$. Let $\boldsymbol{\xi}_{B}^{I} \in \mathbb{R}^{3}$ represent the Cartesian position of the origin of the body fixed frame $o_{B}$ in $\Psi_{I}$, while $\boldsymbol{R}_{B}^{I} \in S O(3)$ represents the orientation of $\Psi_{B}$ with respect to $\Psi_{I}$. Let $\dot{\boldsymbol{\xi}}_{B} \in \mathbb{R}^{3}$ represent the linear velocity vector of the origin of $\Psi_{B}$ with respect to $\Psi_{I}$ expressed in $\Psi_{I}$, while $\boldsymbol{\omega}_{B}^{B, I} \in \mathbb{R}^{3}$ represents the angular velocity vector of $\Psi_{B}$ with respect to $\Psi_{I}$, expressed in $\Psi_{B}$. Let $m$ denote the mass of the vehicle, and $\boldsymbol{J} \in \mathbb{R}^{3 \times 3}$ denote its constant mass moment of inertia matrix expressed in $\Psi_{B}$. The equations of motion of the UAV can be expressed as [20]

$$
\begin{aligned}
& \dot{\boldsymbol{H}}_{B}^{I}=\boldsymbol{H}_{B}^{I} \tilde{\boldsymbol{T}}_{B}^{B, I}, \\
& \mathcal{I}_{B}^{B, I}=\tilde{\boldsymbol{P}}^{B} \boldsymbol{T}_{B}^{B, I}+\boldsymbol{W}_{g}^{B}+\boldsymbol{W}_{c}^{B}-\boldsymbol{W}_{\mathrm{int}}^{B},
\end{aligned}
$$

where $\boldsymbol{H}_{B}^{I} \in S E(3)$ denotes the homogenous matrix from $\Psi_{B}$ to $\Psi_{I}$. The twist $\tilde{\boldsymbol{T}}_{B}^{B, I}$ of $\Psi_{B}$ with respect to $\Psi_{I}$, expressed in $\Psi_{B}$ is defined as

$$
\tilde{\boldsymbol{T}}_{B}^{B, I}:=\left(\begin{array}{cc}
\tilde{\boldsymbol{\omega}}_{B}^{B, I} & \boldsymbol{v}_{B}^{B, I} \\
\mathbf{0} & 0
\end{array}\right):=\left(\begin{array}{cc}
\boldsymbol{R}_{I}^{B} \dot{\boldsymbol{R}}_{B}^{I} & \boldsymbol{R}_{I}^{B} \dot{\boldsymbol{\xi}}_{B}^{I} \\
\mathbf{0} & 0
\end{array}\right) \in \operatorname{se}(3),
$$

which is an element of the Lie algebra of $S E(3)$. The tilde map (operating on $\boldsymbol{\omega}$ ) is defined as $(\cdot)^{\sim}: \mathbb{R}^{3} \rightarrow$ so(3), such that $\tilde{\boldsymbol{\omega}} \boldsymbol{x}=\boldsymbol{\omega} \wedge \boldsymbol{x}, \forall \boldsymbol{x} \in \mathbb{R}^{3}$. The generalized momentum of the body is denoted by $\boldsymbol{P}^{B}:=\mathcal{I}_{B}^{B, I}$ where $\boldsymbol{T}_{B}^{B, I} \in \mathbb{R}^{6}$ denotes the vector of Plücker coordinates of the twist ${ }^{2}$, and $\mathcal{I} \in \mathbb{R}^{6 \times 6}$ denotes the generalized inertia tensor expressed in $\Psi_{B}$ given by

$$
\boldsymbol{T}_{B}^{B, I}=\left(\begin{array}{c}
\boldsymbol{\omega}_{B}^{B, I} \\
\boldsymbol{v}_{B}^{B, I}
\end{array}\right), \quad \mathcal{I}=\left(\begin{array}{cc}
\boldsymbol{J} & \mathbf{0} \\
\mathbf{0} & m \boldsymbol{I}_{3}
\end{array}\right),
$$

where $\boldsymbol{I}_{i}$ denotes the identity matrix of dimension $i$, and $\mathbf{0}$ denotes a matrix of zeros. The skew-symmetric matrix $\tilde{\boldsymbol{P}}^{B}$ is constructed by

$$
\tilde{\boldsymbol{P}}:=\left(\begin{array}{cc}
\tilde{\boldsymbol{P}}_{\omega} & \tilde{\boldsymbol{P}}_{v} \\
\tilde{\boldsymbol{P}}_{v} & 0
\end{array}\right), \quad \boldsymbol{P}=\left(\begin{array}{c}
\boldsymbol{P}_{\omega} \\
\boldsymbol{P}_{v}
\end{array}\right) \in \mathbb{R}^{6} .
$$

Moreover, the wrenches applied to the rigid body include the propellers' control wrench $\boldsymbol{W}_{c}^{B}$, the gravity wrench $\boldsymbol{W}_{g}^{B}$,

\footnotetext{
${ }^{2}$ With an abuse of notation, we call both an element of $\mathfrak{s e}(3)$ and its corresponding vector representation a twist for simplicity, as it will always be clear from the context. The same applies for wrenches.
} 
and the interaction wrench $\boldsymbol{W}_{\text {int }}^{B}$ applied to the UAV's body, expressed in $\Psi_{B}$.

Finally, a change of coordinates of twists is achieved by using the adjoint mapping $\boldsymbol{A} \boldsymbol{d}_{\boldsymbol{H}}: \mathbb{R}^{6} \rightarrow \mathbb{R}^{6}$, given by

$$
\boldsymbol{A d} d_{H}:=\left(\begin{array}{cc}
\boldsymbol{R} & 0 \\
\tilde{\xi} \boldsymbol{R} & \boldsymbol{R}
\end{array}\right), \quad \boldsymbol{H}:=\left(\begin{array}{cc}
\boldsymbol{R} & \boldsymbol{\xi} \\
\mathbf{0} & 1
\end{array}\right),
$$

while wrenches change coordinates by the transpose of the adjoint map.

\section{Port-HAMiltonian CONTROL}

\section{A. UAV's Port-Hamiltonian Model}

In the port-Hamiltonian framework [21], we can rewrite the UAV's equations of motion $(4,5)$ as

$$
\begin{aligned}
\frac{d}{d t}\left(\begin{array}{c}
\boldsymbol{H}_{B}^{I} \\
\boldsymbol{P}^{B}
\end{array}\right) & =\left(\begin{array}{cc}
\mathbf{0} & T \bar{L}_{\boldsymbol{H}_{B}^{I}} \\
-T \bar{L}_{\boldsymbol{H}_{B}^{I}}^{I} & \tilde{\boldsymbol{P}}^{B}
\end{array}\right)\left(\begin{array}{l}
\partial \mathcal{H} / \partial \boldsymbol{H}_{B}^{I} \\
\partial \mathcal{H} / \partial \boldsymbol{P}^{B}
\end{array}\right)+\left(\begin{array}{c}
\mathbf{0} \\
\boldsymbol{I}_{6}
\end{array}\right) \boldsymbol{W}^{B}, \\
\boldsymbol{T}_{B}^{B, I} & =\left(\begin{array}{ll}
\mathbf{0} & \boldsymbol{I}_{6}
\end{array}\right)\left(\begin{array}{l}
\partial \mathcal{H} / \partial \boldsymbol{H}_{B}^{I} \\
\partial \mathcal{H} / \partial \boldsymbol{P}^{B}
\end{array}\right),
\end{aligned}
$$

where the Hamiltonian $\mathcal{H}$ of the system is given only by the kinetic energy of the system, i.e. $\mathcal{H}\left(\boldsymbol{H}_{B}^{I}, \boldsymbol{P}^{B}\right)=$ $\frac{1}{2}\left(\boldsymbol{P}^{B}\right)^{\top} \mathcal{I}^{-1} \boldsymbol{P}^{B}$. Let the map $T L_{\boldsymbol{H}}: \mathfrak{s e}(3) \rightarrow T_{H} S E(3)$ denote the tangent map of the left transport map $L_{H}$ of $S E(3)$, then the map $T \bar{L}_{\boldsymbol{H}}: \mathbb{R}^{6} \rightarrow T_{H} S E(3)$ is defined as the composition of $T L_{\boldsymbol{H}}$ and the tilde map, while $T \bar{L}_{\boldsymbol{H}}^{*}$ is its dual. In other words, $T \bar{L}_{\boldsymbol{H}_{B}^{I}}\left(\boldsymbol{T}_{B}^{B, I}\right) \equiv \boldsymbol{H}_{B}^{I} \tilde{\boldsymbol{T}}_{B}^{B, I}$. Finally, the total wrench applied to the UAV's rigid body is given by

$$
\boldsymbol{W}^{B}=\boldsymbol{W}_{c}^{B}+\boldsymbol{W}_{g}^{B}-\boldsymbol{W}_{\mathrm{int}}^{B},
$$

where the gravitational wrench is assumed to be an external wrench applied to the body rather than stored potential energy ${ }^{3}$.

\section{B. Geometric Impedance Controller}

The impedance controller used in this work is depicted in Fig. 2. It consists of a spatial 6D spring connected between the UAV's end-effector frame ${ }^{4} \Psi_{E}$ and the command frame $\Psi_{C}$, in addition to a damper connected between $\Psi_{B}$ and $\Psi_{I}$.

A spatial spring is a mechanical storage element of potential energy with a displacement being the relative configuration of the two extremes of the spring. This spring is characterized by an energy function $\mathcal{H}_{p}: S E(3) \rightarrow \mathbb{R}$. In one of the possible models of spatial springs [20], [22], the potential energy function $\mathcal{H}_{\text {spr }}\left(\boldsymbol{H}_{E}^{C}\right)$ takes the form

$\mathcal{H}_{\text {spr }}(\boldsymbol{R}, \boldsymbol{\xi})=\frac{1}{4} \boldsymbol{\xi}^{\top} \boldsymbol{K}_{t} \boldsymbol{\xi}+\frac{1}{4} \boldsymbol{\xi}^{\top} \boldsymbol{R} \boldsymbol{K}_{t} \boldsymbol{R}^{\top} \boldsymbol{\xi}-\operatorname{tr}\left(\boldsymbol{G}_{o}\left(\boldsymbol{R}-\boldsymbol{I}_{3}\right)\right)$,

where $\boldsymbol{K}_{t} \in \mathbb{R}^{3 \times 3}$ is the translational stiffness matrix, $\boldsymbol{G}_{o} \in \mathbb{R}^{3 \times 3}$ is the orientational co-stiffness matrix, and $\operatorname{tr}(\cdot)$ denotes the matrix trace.

\footnotetext{
${ }^{3}$ Thus, $\partial \mathcal{H} / \partial \boldsymbol{H}_{B}^{I}=\mathbf{0}$ in equation (10).

${ }^{4}$ It is also a body-fixed frame as shown in Fig. 2
}

For the potential function (12), the components $\tau_{\mathrm{spr}}^{E}, \boldsymbol{f}_{\mathrm{spr}}^{E}$ of the elastic wrench $\boldsymbol{W}_{\mathrm{spr}}^{E}$ that the spatial spring applies to the UAV's end-effector take the form [22]

$$
\begin{aligned}
& \tilde{\boldsymbol{\tau}}_{\mathrm{spr}}^{E}(\boldsymbol{R}, \boldsymbol{\xi})=-2 \operatorname{as}\left(\boldsymbol{G}_{o} \boldsymbol{R}\right)-\operatorname{as}\left(\boldsymbol{G}_{t} \boldsymbol{R}^{\top} \tilde{\boldsymbol{\xi}}^{2} \boldsymbol{R}\right), \\
& \tilde{\boldsymbol{f}}_{\mathrm{spr}}^{E}(\boldsymbol{R}, \boldsymbol{\xi})=-\boldsymbol{R}^{\top} \operatorname{as}\left(\boldsymbol{G}_{t} \tilde{\boldsymbol{\xi}}\right) \boldsymbol{R}-\operatorname{as}\left(\boldsymbol{G}_{t} \boldsymbol{R}^{\top} \tilde{\boldsymbol{\xi}} \boldsymbol{R}\right),
\end{aligned}
$$

where as $(\cdot)$ denotes the skew-symmetric part of a given matrix, i.e. $\operatorname{as}(\boldsymbol{A})=\frac{1}{2}\left(\boldsymbol{A}-\boldsymbol{A}^{\top}\right)$.

Remark 1: The elastic wrench (13) is derived by computing the differential of $\mathcal{H}_{\mathrm{spr}}$ defined as $d \mathcal{H}_{\mathrm{spr}}: S E(3) \rightarrow$ $T^{*} S E(3)$. By using the dual map $T L_{\boldsymbol{H}}^{*}$, we can transform $d \mathcal{H}_{p}$ to an element of $\mathfrak{s e}^{*}(3)$, then using the Plücker coordinates of the wrench we can get an element of $\mathbb{R}^{6}$. Therefore, the elastic wrench can be represented as

$$
\boldsymbol{W}_{\mathrm{spr}}^{E}=-T \bar{L}_{\boldsymbol{H}_{E}^{C}}^{*} d \mathcal{H}_{\mathrm{spr}}\left(\boldsymbol{H}_{E}^{C}\right) .
$$

Spatial damping acts to dissipate the energy stored in the overall system by retarding the motion of the two extremes of the damper. Similar to the spatial springs, it is also possible to define geometrically consistent spatial dampers. However, for quasi-static interaction tasks, linear dampers suffice [23].

For the impedance controller used, the damping wrench applied is given by

$$
\boldsymbol{W}_{\mathrm{dmp}}^{B}=-\boldsymbol{K}_{d} \boldsymbol{T}_{B}^{B, I},
$$

where $\boldsymbol{K}_{d} \in \mathbb{R}^{6 \times 6}$ is a symmetric positive definite matrix. The overall impedance controller is then given by

$$
\boldsymbol{W}_{\mathrm{imp}}^{B}=-\boldsymbol{W}_{g}^{B}+\boldsymbol{A} \boldsymbol{d}_{\boldsymbol{H}_{B}^{E}}^{\top} \boldsymbol{W}_{\mathrm{spr}}^{E}+\boldsymbol{W}_{\mathrm{dmp}}^{B},
$$

where the first term compensates the gravity wrench.

\section{Passivity Analysis I}

The closed-loop system is defined by the Hamiltonian $\mathcal{H}_{\mathrm{cl}}$ which is the sum of the UAV's kinetic energy and the virtual spring's potential energy, given by

$$
\mathcal{H}_{\mathrm{cl}}(\boldsymbol{x})=\frac{1}{2}\left(\boldsymbol{P}^{B}\right)^{\top} \mathcal{I}^{-1} \boldsymbol{P}^{B}+\mathcal{H}_{\mathrm{spr}}\left(\boldsymbol{H}_{E}^{C}\right),
$$

where the state $\boldsymbol{x}=\left(\boldsymbol{H}_{E}^{C}, \boldsymbol{P}^{B}\right) \in S E(3) \times \mathbb{R}^{6}$.

By substituting the geometric impedance control law (16) in the open-loop model (10) and using equations $(14,15)$, we can write the closed-loop system in port-Hamiltonian form as

$$
\dot{\boldsymbol{x}}=[\boldsymbol{J}(\boldsymbol{x})-\boldsymbol{R}] \frac{\partial \mathcal{H}_{\mathrm{cl}}(\boldsymbol{x})}{\partial \boldsymbol{x}}-\boldsymbol{G} \boldsymbol{W}_{\mathrm{int}}^{B},
$$

where the structure matrix $\boldsymbol{J}(\boldsymbol{x})$ is skew-symmetric, the damping matrix $\boldsymbol{R}$ is positive semi-definite, and $\boldsymbol{G}$ is an input matrix. The three matrices are, respectively, given by

$$
\begin{gathered}
\boldsymbol{J}(\boldsymbol{x})=\left(\begin{array}{cc}
\mathbf{0} & T \bar{L}_{\boldsymbol{H}_{E}^{C}} \circ \boldsymbol{A d}_{\boldsymbol{H}_{B}^{E}} \\
-\boldsymbol{A d}_{\boldsymbol{H}_{B}^{E}}^{\top} \circ T \bar{L}_{\boldsymbol{H}_{E}^{C}} & \tilde{\boldsymbol{P}}^{B}
\end{array}\right), \\
\boldsymbol{R}=\left(\begin{array}{cc}
\mathbf{0} & \mathbf{0} \\
\mathbf{0} & \boldsymbol{K}_{d}
\end{array}\right), \quad \boldsymbol{G}=\left(\begin{array}{c}
\mathbf{0} \\
\boldsymbol{I}_{6}
\end{array}\right),
\end{gathered}
$$

where $\circ$ denotes the composition operator. 
The passivity of the system with respect to the interaction power port $\left(\boldsymbol{T}_{B}^{B, I},-\boldsymbol{W}_{\text {int }}^{B}\right)$ can be analyzed by using (17) as a storage function. Accordingly, its time derivative yields

$$
\begin{aligned}
\dot{\mathcal{H}}_{\mathrm{cl}} & =\frac{\partial^{\top} \mathcal{H}_{\mathrm{cl}}}{\partial \boldsymbol{x}} \boldsymbol{J}(\boldsymbol{x}) \frac{\partial \mathcal{H}_{\mathrm{cl}}}{\partial \boldsymbol{x}}-\frac{\partial^{\top} \mathcal{H}_{\mathrm{cl}}}{\partial \boldsymbol{x}} \boldsymbol{R} \frac{\partial \mathcal{H}_{\mathrm{cl}}}{\partial \boldsymbol{x}}-\frac{\partial^{\top} \mathcal{H}_{\mathrm{cl}}}{\partial \boldsymbol{x}} \boldsymbol{G} \boldsymbol{W}_{\mathrm{int}}^{B} \\
& =-\frac{\partial^{\top} \mathcal{H}_{\mathrm{cl}}}{\partial \boldsymbol{P}^{B}} \boldsymbol{K}_{d} \frac{\partial \mathcal{H}_{\mathrm{cl}}}{\partial \boldsymbol{P}^{B}}-\frac{\partial^{\top} \mathcal{H}_{\mathrm{cl}}}{\partial \boldsymbol{P}^{B}} \boldsymbol{W}_{\mathrm{int}}^{B} \leq-\left(\boldsymbol{W}_{\mathrm{int}}^{B}\right)^{\top} \boldsymbol{T}_{B}^{B, I},
\end{aligned}
$$

which follows from the skew-symmetry of $\boldsymbol{J}(\boldsymbol{x})$ and the positive-definiteness of $\boldsymbol{K}_{d}$. Thus, the passivity of the basic geometric impedance controller (16) with respect to the interaction port is concluded from the positive definiteness of (17) and from the inequality in (21). This guarantees the contact stability of the system with any passive arbitrary environment.

\section{ENERGY TANK-BASED WRENCH TRACKING}

\section{A. Wrench Tracking Control Law}

In addition to the impedance controller, presented in the previous section, the desired command wrench $\boldsymbol{W}_{\mathrm{cmd}}^{B}$ to be applied to the environment is achieved via the proportionalintegral ${ }^{5}$ control law

$$
\begin{aligned}
& \boldsymbol{W}_{\mathrm{tr}}^{B}(t)=\boldsymbol{K}_{p, w} \boldsymbol{W}_{\mathrm{err}}(t)+\boldsymbol{K}_{i, w} \int \boldsymbol{W}_{\mathrm{err}}(\varsigma) d \varsigma, \\
& \boldsymbol{W}_{\mathrm{err}}(t)=\hat{\boldsymbol{W}}_{\mathrm{int}}^{B}(t)-\boldsymbol{W}_{\mathrm{cmd}}^{B}(t),
\end{aligned}
$$

where $\boldsymbol{K}_{p, w}, \boldsymbol{K}_{i, w} \in \mathbb{R}^{6 \times 6}$ are positive diagonal matrices of proportional and integral gains, respectively. Instead of using a sensor to feedback the interaction wrench, an estimate $\hat{\boldsymbol{W}}_{\text {int }}^{B}$ is computed by the wrench observer presented next.

\section{B. Interaction Wrench Observer}

Consider the UAV's momentum dynamics (5), which can be rewritten as

$$
\dot{\boldsymbol{P}}^{B}=\underbrace{\tilde{\boldsymbol{P}}^{B} \mathcal{I}^{-1} \boldsymbol{P}^{B}+\boldsymbol{W}_{g}^{B}+\boldsymbol{W}_{c}^{B}}_{\boldsymbol{f}\left(\boldsymbol{P}^{B}, \boldsymbol{W}_{c}^{B}\right)}-\boldsymbol{W}_{\mathrm{int}}^{B} .
$$

Similar to [24]-[26], a constant wrench observer is designed as

$$
\begin{aligned}
\hat{\boldsymbol{W}}_{\mathrm{int}}^{B} & =\boldsymbol{K}_{o}\left(\boldsymbol{P}^{B}-\hat{\boldsymbol{P}}\right), \\
\dot{\hat{\boldsymbol{P}}} & =\boldsymbol{f}\left(\boldsymbol{P}^{B}, \boldsymbol{W}_{c}^{B}\right)+\boldsymbol{K}_{o}\left(\boldsymbol{P}^{B}-\hat{\boldsymbol{P}}\right),
\end{aligned}
$$

where $\hat{\boldsymbol{P}} \in \mathbb{R}^{6}$ is an estimate of the actual body momentum, and $\boldsymbol{K}_{o} \in \mathbb{R}^{6 \times 6}$ is a positive diagonal matrix of observer gains.

The relation between the actual and estimated interaction wrench can be derived by substituting $(23,25)$ into the time derivative of (24) which yields

$$
\dot{\hat{\boldsymbol{W}}}_{\mathrm{int}}^{B}+\boldsymbol{K}_{o} \hat{\boldsymbol{W}}_{\mathrm{int}}^{B}=-\boldsymbol{K}_{o} \boldsymbol{W}_{\mathrm{int}}^{B},
$$

which represents six, first-order filters for each component of the interaction wrench.

\footnotetext{
${ }^{5}$ A proportional-integral-derivative control law would require an estimate of the interaction wrench's derivative, which can be acquired by using higher-order observers.
}

Remark 2: In practice, the actual momentum dynamics (23) includes other external wrenches e.g. aerodynamic disturbances, unmodeled dynamics, and parametric uncertainties. The wrench computed from the observer $(24,25)$ will actually be an estimate of all the external disturbances of the system. The separation of the interaction wrench from the other disturbances is a topic of future investigation.

\section{Passivity Analysis II}

The overall observer-based wrench/impedance controller is given by equations $(16,22,24,25)$ :

$$
\boldsymbol{W}_{c}^{B}=\boldsymbol{W}_{\mathrm{imp}}^{B}+\boldsymbol{W}_{\mathrm{tr}}^{B} .
$$

To show that the closed-loop system's passivity is no longer guaranteed after the addition of the observer-based wrench controller, we consider its port-Hamiltonian formulation

$$
\begin{aligned}
\left(\begin{array}{c}
\dot{\boldsymbol{x}} \\
\dot{\hat{\boldsymbol{P}}}
\end{array}\right)= & \left(\begin{array}{cc}
\boldsymbol{J}(\boldsymbol{x})-\boldsymbol{R} & \mathbf{0} \\
\mathbf{0} & -\boldsymbol{I}_{6}
\end{array}\right)\left(\begin{array}{c}
\partial \overline{\mathcal{H}}_{\mathrm{cl}} / \partial \boldsymbol{x} \\
\partial \overline{\mathcal{H}}_{\mathrm{cl}} / \partial \hat{\boldsymbol{P}}
\end{array}\right)-\left(\begin{array}{c}
\boldsymbol{G} \\
\mathbf{0}
\end{array}\right) \boldsymbol{W}_{\mathrm{int}}^{B} \\
& +\left(\begin{array}{c}
\boldsymbol{G} \\
\mathbf{0}
\end{array}\right) \overline{\boldsymbol{\omega}}_{1}(\overline{\boldsymbol{x}}, t)+\left(\begin{array}{c}
\mathbf{0} \\
\boldsymbol{I}_{6}
\end{array}\right) \overline{\boldsymbol{\omega}}_{2}(\overline{\boldsymbol{x}}, t),
\end{aligned}
$$

where the closed-loop Hamiltonian $\overline{\mathcal{H}}_{\mathrm{cl}}$ is given by

$$
\overline{\mathcal{H}}_{\mathrm{cl}}(\overline{\boldsymbol{x}})=\mathcal{H}_{\mathrm{cl}}(\boldsymbol{x})+\mathcal{H}_{\mathrm{obs}}(\hat{\boldsymbol{P}})=\mathcal{H}_{\mathrm{cl}}(\boldsymbol{x})+\frac{1}{2} \hat{\boldsymbol{P}}^{\top} \boldsymbol{K}_{o} \hat{\boldsymbol{P}},
$$

with $\overline{\boldsymbol{x}}=\left(\boldsymbol{H}_{E}^{C}, \boldsymbol{P}^{B}, \hat{\boldsymbol{P}}\right)$, and $\overline{\boldsymbol{\omega}}_{1}, \overline{\boldsymbol{\omega}}_{2} \in \mathbb{R}^{6}$ are given by

$\overline{\boldsymbol{\omega}}_{1}(\overline{\boldsymbol{x}}, t)=\boldsymbol{W}_{\mathrm{tr}}^{B}(t), \quad \overline{\boldsymbol{\omega}}_{2}(\overline{\boldsymbol{x}}, t)=\boldsymbol{f}\left(\boldsymbol{P}^{B}, \boldsymbol{W}_{c}^{B}\right)+\boldsymbol{K}_{o} \boldsymbol{P}^{B}$.

To evaluate the passivity of the system, we consider the time-derivative of $\overline{\mathcal{H}}_{\mathrm{cl}}$ which can be written as

$$
\begin{aligned}
\dot{\overline{\mathcal{H}}}_{\mathrm{cl}}= & -\frac{\partial^{\top} \overline{\mathcal{H}}_{\mathrm{cl}}}{\partial \boldsymbol{P}^{B}} \boldsymbol{K}_{d} \frac{\partial \overline{\mathcal{H}}_{\mathrm{cl}}}{\partial \boldsymbol{P}^{B}}-\frac{\partial^{\top} \overline{\mathcal{H}}_{\mathrm{cl}}}{\partial \hat{\boldsymbol{P}}} \frac{\partial \overline{\mathcal{H}}_{\mathrm{cl}}}{\partial \hat{\boldsymbol{P}}}-\frac{\partial^{\top} \overline{\mathcal{H}}_{\mathrm{cl}}}{\partial \boldsymbol{P}^{B}} \boldsymbol{W}_{\mathrm{int}}^{B} \\
& +\frac{\partial^{\top} \overline{\mathcal{H}}_{\mathrm{cl}}}{\partial \boldsymbol{P}^{B}} \overline{\boldsymbol{\omega}}_{1}+\frac{\partial^{\top} \overline{\mathcal{H}}_{\mathrm{cl}}}{\partial \hat{\boldsymbol{P}}} \overline{\boldsymbol{\omega}}_{2} .
\end{aligned}
$$

Due to the sign indefiniteness of the last two terms in the previous equation, the passivity of the system is no longer guaranteed. Next, we show how energy tanks can be exploited to restore the passivity of the closed-loop system.

\section{Energy Tank Augmentation}

As evident in (28), the two variables $\overline{\boldsymbol{\omega}}_{1}, \overline{\boldsymbol{\omega}}_{2}$ are considered to be extra inputs to the system which can inject energy and potentially violate its passivity. In the energy balance (31), the first two terms represent the actively damped energy by the system:

$$
\boldsymbol{D}_{1}\left(\boldsymbol{P}^{B}\right):=\frac{\partial^{\top} \overline{\mathcal{H}}_{\mathrm{cl}}}{\partial \boldsymbol{P}^{B}} \boldsymbol{K}_{d} \frac{\partial \overline{\mathcal{H}}_{\mathrm{cl}}}{\partial \boldsymbol{P}^{B}}, \quad \boldsymbol{D}_{2}(\hat{\boldsymbol{P}}):=\frac{\partial^{\top} \overline{\mathcal{H}}_{\mathrm{cl}}}{\partial \hat{\boldsymbol{P}}} \frac{\partial \overline{\mathcal{H}}_{\mathrm{cl}}}{\partial \hat{\boldsymbol{P}}} .
$$

Energy tanks, exploit this dissipated energy by routing it to a virtual energy tank, and then reusing this energy to implement the desired control actions while preserving the overall system's passivity.

To add an energy-tank to the observer-based controller discussed earlier, we augment $\overline{\boldsymbol{x}}$ by a new state $x_{t} \in \mathbb{R}$ 
representing the state of the energy tank. The augmented port-Hamiltonian system is then given by

$$
\begin{aligned}
\dot{\overline{\boldsymbol{x}}}= & \left(\begin{array}{cc}
\boldsymbol{J}(\boldsymbol{x})-\boldsymbol{R} & \mathbf{0} \\
\mathbf{0} & -\boldsymbol{I}_{6}
\end{array}\right) \frac{\partial \overline{\mathcal{H}}_{\mathrm{cl}}}{\partial \overline{\boldsymbol{x}}}-\left(\begin{array}{c}
\boldsymbol{G} \\
\mathbf{0}
\end{array}\right) \boldsymbol{W}_{\mathrm{int}}^{B} \\
& +\left(\begin{array}{c}
\boldsymbol{G} \\
\mathbf{0}
\end{array}\right) \boldsymbol{\omega}_{1}+\left(\begin{array}{c}
\mathbf{0} \\
\boldsymbol{I}_{6}
\end{array}\right) \boldsymbol{\omega}_{2}, \\
\dot{x}_{t}= & \frac{\beta}{x_{t}}\left(\eta_{1} \boldsymbol{D}_{1}+\eta_{2} \boldsymbol{D}_{2}\right)+u_{t},
\end{aligned}
$$

where $u_{t} \in \mathbb{R}$ is the control input of the tank, $\beta$ is defined as

$$
\beta= \begin{cases}1 & \text { if } E_{t} \leq E_{t}^{+} \\ 0 & \text { otherwise }\end{cases}
$$

where $E_{t}:=\frac{1}{2} x_{t}^{2}$ is the associated tank energy, and $E_{t}^{+} \in$ $\mathbb{R}^{+}$is an upper bound on the energy that can be stored in the tank. This upper bound represents the allocated energy budget to perform the required interaction task. The two variables $0<\eta_{i} \leq 1$, for $i \in\{1,2\}$, control the amount of actively damped energy directed to the energy tank, where $\eta_{i}=1$ means that all the damped energy is directed to the tank. The two new inputs $\boldsymbol{\omega}_{i} \in \mathbb{R}^{6}$, for $i \in\{1,2\}$, are given by

$$
\boldsymbol{\omega}_{i}=\alpha \overline{\boldsymbol{\omega}}_{i}, \quad \alpha= \begin{cases}1 & \text { if } E_{t} \geq E_{t}^{-} \\ 0 & \text { otherwise }\end{cases}
$$

where $\alpha$ is responsible for detaching the energy tank if the lower limit $E_{t}^{-} \in \mathbb{R}^{+}$is reached.

The energy tank input $u_{t}$ is chosen to be

$$
u_{t}=-\frac{1}{x_{t}}\left(\boldsymbol{\omega}_{1}^{\top} \frac{\partial \overline{\mathcal{H}}_{\mathrm{cl}}}{\partial \boldsymbol{P}^{B}}+\boldsymbol{\omega}_{2}^{\top} \frac{\partial \overline{\mathcal{H}}_{\mathrm{cl}}}{\partial \hat{\boldsymbol{P}}}\right) .
$$

This choice guarantees that the connection between the energy tank and the observer-based wrench controller is a power-preserving Dirac structure [18].

Finally, the overall energy tank-based controller consists of the control law (27) with the energy tank dynamics (34). The wrench tracking control law and the observer dynamics are now, respectively, computed by

$$
\boldsymbol{W}_{\mathrm{tr}}^{B}=\boldsymbol{\omega}_{1}, \quad \dot{\hat{\boldsymbol{P}}}=-\boldsymbol{K}_{o} \hat{\boldsymbol{P}}+\boldsymbol{\omega}_{2} .
$$

\section{E. Passivity Analysis III}

To conclude the passivity of the augmented closed-loop system, we consider as a storage function the total energy

$$
\mathcal{H}_{a}\left(\boldsymbol{x}_{a}\right)=\mathcal{H}_{\mathrm{cl}}(\boldsymbol{x})+\mathcal{H}_{\mathrm{obs}}(\hat{\boldsymbol{P}})+E_{t}\left(x_{t}\right),
$$

where $\boldsymbol{x}_{a}=\left(\boldsymbol{x}, \hat{\boldsymbol{P}}, x_{t}\right)$. Using equation (37), the augmented port-Hamiltonian model $(33,34)$ can be rewritten as

$$
\dot{\boldsymbol{x}}_{a}=\left[\overline{\boldsymbol{J}}\left(\boldsymbol{x}_{\boldsymbol{a}}\right)-\overline{\boldsymbol{R}}\left(\boldsymbol{x}_{\boldsymbol{a}}\right)\right] \frac{\partial \mathcal{H}_{\mathrm{aug}}}{\partial \boldsymbol{x}_{\mathrm{aug}}}-\overline{\boldsymbol{G}} \boldsymbol{W}_{\mathrm{int}}^{B},
$$

where The three matrices $\overline{\boldsymbol{J}}, \overline{\boldsymbol{R}}, \overline{\boldsymbol{G}}$ are, respectively, given by

$$
\overline{\boldsymbol{J}}\left(\boldsymbol{x}_{\boldsymbol{a}}\right)=\left(\begin{array}{ccc}
J(\boldsymbol{x}) & \mathbf{0} & \frac{G \omega_{1}}{x_{t}} \\
\mathbf{0} & \mathbf{0} & \frac{\boldsymbol{\omega}_{2}}{x_{t}} \\
-\frac{\boldsymbol{\omega}_{1}^{\top} \boldsymbol{G}^{\top}}{x_{t}} & -\frac{\boldsymbol{\omega}_{2}^{\top}}{x_{t}} & \mathbf{0}
\end{array}\right),
$$

$$
\overline{\boldsymbol{R}}\left(\boldsymbol{x}_{\boldsymbol{a}}\right)=\left(\begin{array}{ccc}
\boldsymbol{R} & \mathbf{0} & \mathbf{0} \\
\mathbf{0} & \boldsymbol{I}_{6} & \mathbf{0} \\
-\frac{\beta \eta_{1} \overline{\boldsymbol{D}}_{1}}{x_{t}} & -\frac{\beta \eta_{2} \overline{\boldsymbol{D}}_{2}}{x_{t}} & \mathbf{0}
\end{array}\right), \quad \overline{\boldsymbol{G}}=\left(\begin{array}{c}
\boldsymbol{G} \\
\mathbf{0} \\
\mathbf{0}
\end{array}\right)
$$

with $\overline{\boldsymbol{D}}_{1}, \overline{\boldsymbol{D}}_{2}$ defined such that

$$
\overline{\boldsymbol{D}}_{1} \frac{\partial \mathcal{H}_{a}}{\partial \boldsymbol{x}}:=\boldsymbol{D}_{1}, \quad \overline{\boldsymbol{D}}_{2}:=\frac{\partial^{\top} \mathcal{H}_{a}}{\partial \hat{\boldsymbol{P}}} .
$$

Similar to the previous analysis, the time derivative of $\mathcal{H}_{a}$ is evaluated along the augmented closed loop dynamics (40) to assess the system's passivity. Due to the skew-symmetry of $\overline{\boldsymbol{J}}$, the time derivative of $\mathcal{H}_{a}$ reduces to

$$
\begin{aligned}
\dot{\mathcal{H}}_{a} & =-\boldsymbol{D}_{1}-\frac{\partial^{\top} \overline{\mathcal{H}}_{a}}{\partial \boldsymbol{P}^{B}} \boldsymbol{W}_{\mathrm{int}}^{B}-\boldsymbol{D}_{2}+\beta \eta_{1} \boldsymbol{D}_{1}+\beta \eta_{2} \boldsymbol{D}_{2}, \\
& =-\left(1-\beta \eta_{1}\right) \boldsymbol{D}_{1}-\left(1-\beta \eta_{2}\right) \boldsymbol{D}_{2}-\frac{\partial^{\top} \overline{\mathcal{H}}_{a}}{\partial \boldsymbol{P}^{B}} \boldsymbol{W}_{\mathrm{int}}^{B}, \\
& \leq-\left(\boldsymbol{W}_{\mathrm{int}}^{B}\right)^{\top} \boldsymbol{T}_{B}^{B, I},
\end{aligned}
$$

which follows from the fact that $\beta \in\{0,1\}, 0<\eta_{i} \leq 1$, and $\boldsymbol{D}_{i} \geq 0$ for $i \in\{1,2\}$. Hence, the system's contact stability with respect to any passive arbitrary environment is restored by the addition of the energy tank.

\section{Simulation Results}

The proposed energy tank-based control system has been applied to a fully-actuated hexarotor simulated in 20Sim [27]. The goal of the presented simulation results is to show the passivity of the system that is restored by the inclusion of the energy tank in the control system. This is asserted if the difference between the total energy in the system (39) and the energy injected externally by the environment is nonincreasing, as in the last inequality in (44).

The simulation scenario consists of the aerial robot applying a desired wrench normal to the surface of a compliant vertical wall. The translational $x$ component of the wrench (normal to the surface in contact) is displayed in Fig. 3. The aerial robot starts from a stationary initial configuration and is moved with the basic impedance controller to the vicinity of the wall (phase 1). Afterwards, the wrench tracking controller is turned on with a constant desired force of $10 \mathrm{~N}$ to be applied to the wall (phase 2). Finally, a sinusoidal force profile is commanded (phase 3 ).

Without the energy tanks, it can be seen in the upper diagram in Fig. 4 that there are time periods (shaded area) of active energy generation. This active interval is avoided when energy tanks are used (lower diagram). In the lower diagram it is also shown that the system's strict passivity can be assured by transferring part of the virtual damper's energy (with $\eta_{1}=0.5$ ) to the tank.

\section{EXPERIMENTAL VALIDATION}

The proposed approach has been validated experimentally on a fully-actuated hexarotor that was developed in-house based on off-the-shelf components. The nominal total mass is $m=1.6 \mathrm{~kg}$ and the propulsion system provides a maximum thrust of $16 \mathrm{~N}$ per rotor. The proposed interaction controller was implemented on a Linux PC (running ROS) and interfacing with the onboard Pixhawk flight controller (running 


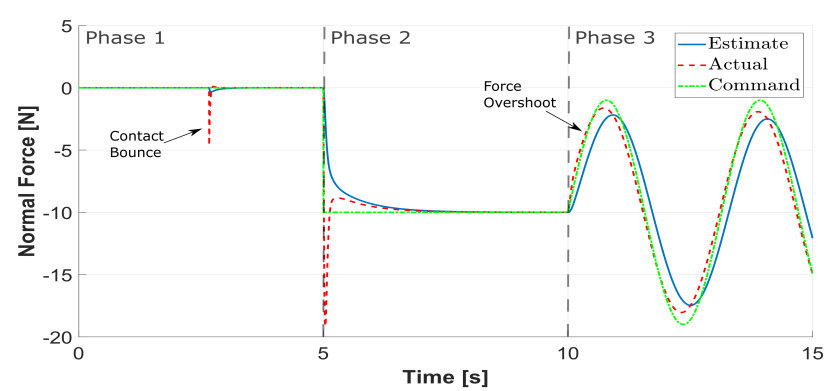

Fig. 3: Simulation: The actual, estimate, and command interaction force applied to the UAV, normal to the surface of the wall.

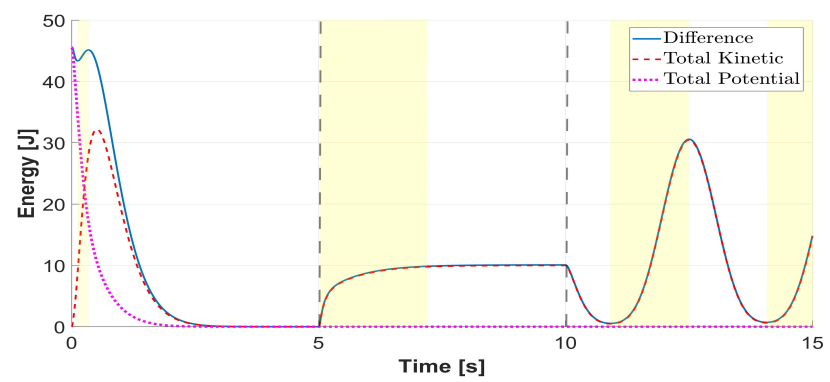

(a) Without Energy Tank

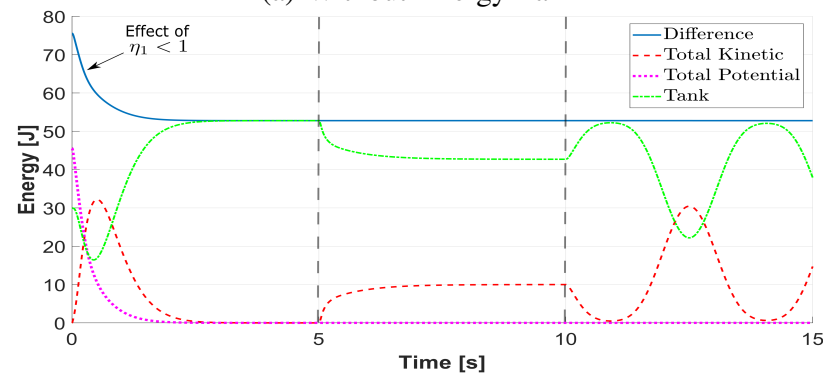

(b) With Energy Tank $\eta_{1}=0.5, \eta_{2}=1$

Fig. 4: Simulation: Comparison of the energies in the system. Regions of active generation are shaded in the upper diagram.

the PX4 software [28]) via serial USB communication at a rate of $100 \mathrm{~Hz}$. Using a multi-sensor fusion algorithm [29], the inertial measurements of the UAV are fused with a motion capture system to provide reliable estimates of the configuration and twist.

The results of an interaction experiment are shown in Figs. 5 and 6. An ATI mini40 force/torque sensor (ATI Industrial Automation) was used to provide ground-truth measurements of the interaction wrench. The experiment scenario consists of the UAV taking off and approaching the force/torque sensor with the basic impedance controller (phase 1), then establishing contact without wrench regulation (phase 2). Then, the wrench regulator is turned on (phase 3 ), and the commanded normal force increases in steps until it reaches 8 $\mathrm{N}$. It can be seen that the wrench observer provides reliable estimates of the interaction wrench, which resulted in a root-mean-squared (RMS) error (between ground-truth and commanded force) of approximately $0.3 \mathrm{~N}$. Power has been supplied to the UAV in the experiments using a power cable,

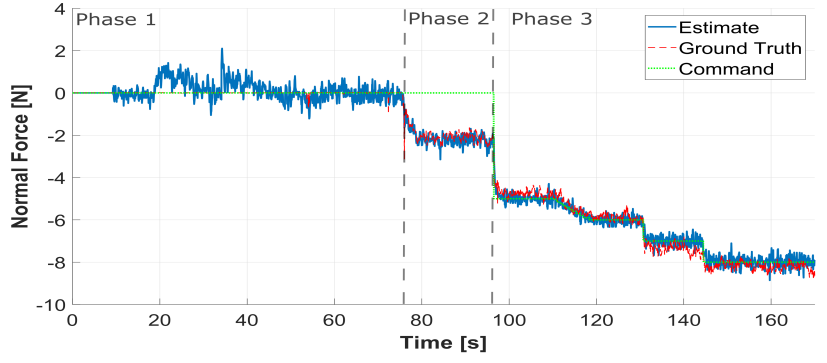

Fig. 5: Experiment: The estimated and tracking error of the interaction forces normal to the surface of the wall.

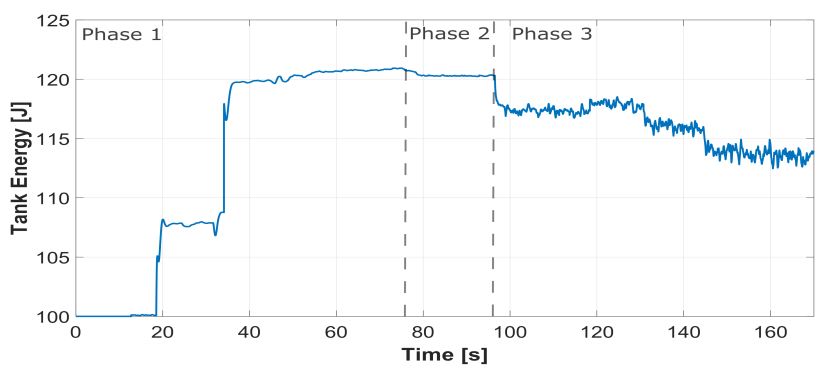

Fig. 6: Experiment: Energy stored in the virtual tank, initialized with $100 \mathrm{~J}$

which as expected adds some constant disturbances to the UAV during flight (as can be seen in phase 1 in Fig. 5). In Fig. 6, it can be seen that the energy is stored in the tank during phases 1 and 2 due to the damper's energy transfered to the tank, which is then used in phase 3 to perform the non-passive action of the wrench regulation.

An interesting behavior that was witnessed during the experiment is that when the commanded force reaches $8 \mathrm{~N}$, one of the UAV's rotors switches off. This is due to the fact that the thrust combination to achieve a normal force of 8 $\mathrm{N}$ requires one of the rotors to be zero. Thanks to the controller's robustness to uncertainties, this input saturation does not destabilize the system. This behavior can be seen in the supplementary video, along with more experiments showing sliding on a smooth surface with different orientations and controlling the UAV's orientation while maintaining contact.

\section{CONCLUSION}

In this paper an energy-aware approach for modeling, analysis, and control of an interactive UAV was presented. The interaction controller was able to simultaneously control the impedance behavior and the interaction wrench while maintaining passivity in a geometrically consistent manner.

The merits of the presented framework are: 1) the importance of the energy and interconnection structure of the system is underscored; 2) the nonlinear geometric structure of rigid body dynamics is exploited in the controller design; 3 ) the passivity of the system enables stable interaction with any arbitrary passive environment; and 4) its passivity-based nature makes the controller robust to parametric uncertainties and modeling errors. 


\section{REFERENCES}

[1] M. Ryll, G. Muscio, F. Pierri, E. Cataldi, G. Antonelli, F. Caccavale, and A. Franchi, "6d physical interaction with a fully actuated aerial robot," in Robotics and Automation (ICRA), 2017 IEEE International Conference on. IEEE, 2017, pp. 5190-5195.

[2] S. Park, J. Her, J. Kim, and D. Lee, "Design, modeling and control of omni-directional aerial robot," in 2016 IEEE/RSJ Int. Conf. Intell. Robot. Syst. IEEE, oct 2016, pp. 1570-1575.

[3] S. Park, J. Lee, J. Ahn, M. Kim, J. Her, G.-H. Yang, and D. Lee, "Odar: Aerial manipulation platform enabling omnidirectional wrench generation," IEEE/ASME Transactions on Mechatronics, vol. 23, no. 4, pp. 1907-1918, 2018.

[4] G. Jiang, R. M. Voyles, and J. J. Choi, "Precision fully-actuated uav for visual and physical inspection of structures for nuclear decommissioning and search and rescue," in 2018 IEEE International Symposium on Safety, Security, and Rescue Robotics (SSRR). IEEE, 2018, pp. $1-7$.

[5] A. Suarez, G. Heredia, and A. Ollero, "Physical-Virtual Impedance Control in Ultralightweight and Compliant Dual-Arm Aerial Manipulators," IEEE Robot. Autom. Lett., vol. 3, no. 3, pp. 2553-2560, jul 2018.

[6] V. Lippiello, G. A. Fontanelli, and F. Ruggiero, "Image-Based VisualImpedance Control of a Dual-Arm Aerial Manipulator," IEEE Robot. Autom. Lett., vol. 3, no. 3, pp. 1856-1863, jul 2018.

[7] J. Duffy, "The fallacy of modern hybrid control theory that is based on orthogonal complements of twist and wrench spaces," Journal of Robotic Systems, vol. 7, no. 2, pp. 139-144, 1990.

[8] C. Schindlbeck and S. Haddadin, "Unified passivity-based cartesian force/impedance control for rigid and flexible joint robots via taskenergy tanks," in Robotics and Automation (ICRA), 2015 IEEE International Conference on. IEEE, 2015, pp. 440-447.

[9] M. Franken, S. Stramigioli, S. Misra, C. Secchi, and A. Macchelli, "Bilateral telemanipulation with time delays: A two-layer approach combining passivity and transparency," IEEE transactions on robotics, vol. 27, no. 4, pp. 741-756, 2011.

[10] C. Secchi, A. Franchi, H. H. Bülthoff, and P. R. Giordano, "Bilateral teleoperation of a group of uavs with communication delays and switching topology," in Robotics and Automation (ICRA), 2012 IEEE International Conference on. IEEE, 2012, pp. 4307-4314.

[11] F. Ferraguti, C. Secchi, and C. Fantuzzi, "A tank-based approach to impedance control with variable stiffness," in Robotics and Automation (ICRA), 2013 IEEE International Conference on. IEEE, 2013, pp. 4948-4953.

[12] F. Ferraguti, N. Preda, A. Manurung, M. Bonfe, O. Lambercy, R. Gassert, R. Muradore, P. Fiorini, and C. Secchi, "An energy tankbased interactive control architecture for autonomous and teleoperated robotic surgery," IEEE Transactions on Robotics, vol. 31, no. 5, pp. 1073-1088, 2015.

[13] K. Kronander and A. Billard, "Passive interaction control with dynamical systems," IEEE Robotics and Automation Letters, vol. 1, no. 1, pp. 106-113, 2016.

[14] A. Dietrich, X. Wu, K. Bussmann, C. Ott, A. Albu-Schaffer, and S. Stramigioli, "Passive Hierarchical Impedance Control Via Energy Tanks," IEEE Robot. Autom. Lett., vol. 2, no. 2, pp. 522-529, apr 2017.

[15] G. Raiola, C. A. Cardenas, T. S. Tadele, T. de Vries, and S. Stramigioli, "Development of a safety-and energy-aware impedance controller for collaborative robots," IEEE Robotics and Automation Letters, vol. 3, no. 2, pp. 1237-1244, 2018.

[16] E. Shahriari, L. Johannsmeier, and S. Haddadin, "Valve-based virtual energy tanks: A framework to simultaneously passify controls and embed control objectives," in 2018 Annual American Control Conference (ACC). IEEE, 2018, pp. 3634-3641.

[17] V. Duindam and S. Stramigioli, "Port-based asymptotic curve tracking for mechanical systems," European Journal of Control, vol. 10, no. 5, pp. 411-420, 2004.

[18] A. Van der Schaft, L2-gain and passivity techniques in nonlinear control. Springer, 2000, vol. 2.

[19] S. Stramigioli, "Energy-aware robotics," in Mathematical Control Theory I. Springer, 2015, pp. 37-50.

[20] S. Stramigioli and V. Duindam, "Variable spatial springs for robot control applications," IEEE/RSJ Int. Conf. Intell. Robot. Syst., vol. 4, pp. 1906-1911, 2001.
[21] A. Van Der Schaft and B. Maschke, "Port-hamiltonian systems on graphs," SIAM Journal on Control and Optimization, vol. 51, no. 2, pp. 906-937, 2013.

[22] S. Stramigioli, Modeling and IPC control of interactive mechanical system - A coordinate-free approach. Springer-Verlag London, 2001.

[23] E. Fasse and J. Broenink, "A spatial impedance controller for robotic manipulation," IEEE Trans. Robot. Autom., vol. 13, no. 4, pp. 546556, 1997.

[24] A. De Luca and R. Mattone, "Sensorless robot collision detection and hybrid force/motion control," in Robotics and Automation, 2005. ICRA 2005. Proceedings of the 2005 IEEE International Conference on. IEEE, 2005, pp. 999-1004.

[25] A. De Luca, A. Albu-Schaffer, S. Haddadin, and G. Hirzinger, "Collision detection and safe reaction with the dlr-iii lightweight manipulator arm," in Intelligent Robots and Systems, 2006 IEEE/RSJ International Conference on. IEEE, 2006, pp. 1623-1630.

[26] K.-S. Kim, K.-H. Rew, and S. Kim, "Disturbance observer for estimating higher order disturbances in time series expansion," IEEE Transactions on automatic control, vol. 55, no. 8, pp. 1905-1911, 2010.

[27] "20Sim 4.7, Controllab Products B.V." [Online]. Available: http://www.20sim.com/

[28] L. Meier, D. Honegger, and M. Pollefeys, "Px4: A node-based multithreaded open source robotics framework for deeply embedded platforms," in Robotics and Automation (ICRA), 2015 IEEE International Conference on. IEEE, 2015, pp. 6235-6240.

[29] S. Lynen, M. Achtelik, S. Weiss, M. Chli, and R. Siegwart, "A robust and modular multi-sensor fusion approach applied to mav navigation," in Proc. of the IEEE/RSJ Conference on Intelligent Robots and Systems (IROS), 2013. 